Supporting Material

for

\title{
Parity and Heteroatom Effects on Open-Shell Exchange Interactions: Nitrenophenyl Iminoylnitroxides
}

\author{
Patrick Taylor, Paul R. Serwinski, Paul M. Lahti* \\ Department of Chemistry, University of Massachusetts, Amherst, MA 01003 USA
}

- Experimental details

Page S1

- Figure S1. UB3LYP/6-31G*//UB3LYP/6-31G* energies,

Page S2 selected bond lengths (angstroms), and Mulliken spin density populations for quartet and doublet states of models for pNPhIN and mNPhIN.

- Computed spin densities for models to pNPhIN and mNPhIN

Pages S3-S6

- TD-DFT/UB3LYP/6-31G* UV-vis computed transitions 


\section{Experimental.}

UV-vis spectral studies. For cryogenic UV-vis studies, the radical precursors were dissolved in 2-methyltetrahydrofuran (MTHF, $\sim 2 \mathrm{mg} / 1 \mathrm{~mL}$ ), placed in a copper sample holder, prefrozen at $77 \mathrm{~K}$, placed under vacuum, and cooled to 14-20 K using an APD Cryogenics Displex CS-202 close-cycle circulating helium cryostat. For each sample, a pre-photolysis UV-vis spectrum was obtained at low temperature. The sample was then photolyzed for 3-5 min at $350 \mathrm{~nm}$ using a Rayonet carousel photoreactor and maintaining the low temperature, and the UV-vis spectrum again obtained.

ESR spectral studies. For ESR spectroscopic studies, the radical precursors were dissolved in MTHF in $4 \mathrm{~mm}$ o.d. quartz tubes, subjected to thorough freeze pump thaw degassing, sealed under helium, placed in the spectrometer cavity of a Bruker ESP-300E spectrometer with precooling to $4.2 \mathrm{~K}$ using an Oxford Instruments ESR-9000 cryostat, then photolyzed in situ through an Oriel 77557 liquid light guide using a water-filtered $1000 \mathrm{~W}$ Xenon arc lamp. ESR spectra were also obtained by freezing degassed MTHF samples of radical precursors at $77 \mathrm{~K}$ in a quartz finger dewar with LN2, then photolyzing with a Rayonet carousel photoreactor using $350 \mathrm{~nm}$ bulbs.

Synthesis of pN3PhIN. To a stirred solution of pN3PhNN $(0.100 \mathrm{~g}, 0.365 \mathrm{mmol})$ in $25 \mathrm{~mL}$ of dichloromethane (DCM) under argon was added $\mathrm{NaNO}_{2}(0.500 \mathrm{~g}, 7.25 \mathrm{mmol})$ and $0.5 \mathrm{~mL}$ of a $0.1 \mathrm{~N} \mathrm{HCl}$ solution. The reaction was stirred $30 \mathrm{~min}$ then $5 \mathrm{~mL}$ of water was added and the organic layer collected, back extracting the aqueous phase with additional DCM ( 3 x $15 \mathrm{~mL})$. The combined organic layers were dried with $\mathrm{Na}_{2} \mathrm{CO}_{3}$, filtered and concentrated under reduced pressure to give a red solid. The crude product was purified by column chromatography (silica, 7:3 hexanes:ethylacetate) to afford pN3PhIN as a dark red solid $\left(0.071 \mathrm{~g}, 76 \%, \mathrm{mp} 59-61{ }^{\circ} \mathrm{C}\right)$. HR-MS (EI): Calc. $\mathrm{C}_{13} \mathrm{H}_{16} \mathrm{~N}_{5} \mathrm{O} \mathrm{m} / \mathrm{z}=268.1355$; found $\mathrm{m} / \mathrm{z}=258.1332 *{ }^{*}$ FTIR $\left(\mathrm{KBr}, \mathrm{cm}^{-1}\right)$ : 3067, 2980, 2115 (strong, $\mathrm{N}_{3}$ str). ESR (toluene, $9.650 \mathrm{GHz}$ ): $\mathrm{a}_{\mathrm{N}}=9.00,4.21 \mathrm{G}$.

Synthesis of mN3PhIN. To a stirred solution of $\mathbf{m N 3 P h N N}(0.025 \mathrm{~g}, 0.091 \mathrm{mmol})$ in $20 \mathrm{~mL}$ of dichloromethane (DCM) under argon was added $\mathrm{NaNO}_{2}(0.126 \mathrm{~g}, 1.82 \mathrm{mmol})$ and $0.5 \mathrm{~mL}$ of a $0.1 \mathrm{~N} \mathrm{HCl}$ solution. The reaction was stirred $15 \mathrm{~min}$ then $2 \mathrm{~mL}$ of water was added and the organic layer collected, back extracting the aqueous phase with additional DCM (2 x $10 \mathrm{~mL})$. The combined organic layers were dried with $\mathrm{Na}_{2} \mathrm{CO}_{3}$, filtered and concentrated under reduced pressure to give a red oil. The crude product was purified by column chromatography (silica, 7:3 hexanes:ethylacetate) to afford $\mathbf{m N 3 P h I N}$ as a dark red oil. HR-MS (EI): Calc. $\mathrm{C}_{13} \mathrm{H}_{16} \mathrm{~N}_{5} \mathrm{O} \mathrm{m} / \mathrm{z}=$ 268.1355; found $\mathrm{m} / \mathrm{z}=258.1353 . *$ FTIR $\left(\mathrm{KBr}, \mathrm{cm}^{-1}\right): 3085,2981,2131$ (strong, $\mathrm{N}_{3}$ str). ESR (toluene, $9.633 \mathrm{GHz}$ ): $\mathrm{a}_{\mathrm{N}}=8.84,4.31 \mathrm{G}$.

\footnotetext{
* The authors thank the UMass-Amherst Mass Spectrometry and Molecular Weight Determination Facility for mass spectral analyses.
} 


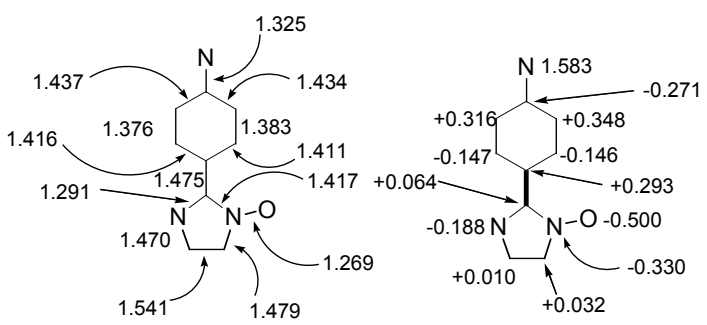

${ }^{2} \mathrm{~A}_{1}$ state, UB3LYP/6-31G* optimized $E=-587.0846075\left(<S^{2}>=1.80\right)$ $+2.1 \mathrm{kcal} / \mathrm{mol}$

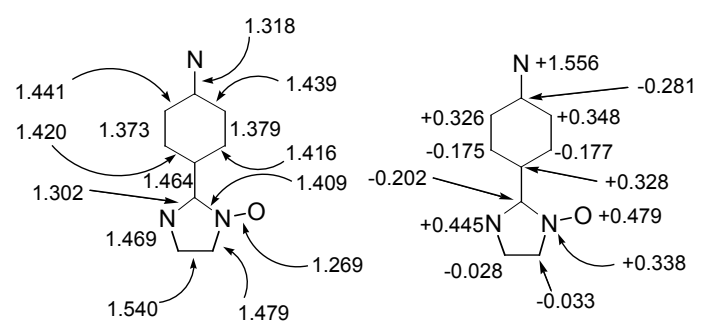

${ }^{4} \mathrm{~A}_{1}$ state, UB3LYP/6-31G* optimized $E=-587.088008\left(<S^{2}>=3.84\right)$ $0.0 \mathrm{kcal} / \mathrm{mol}$
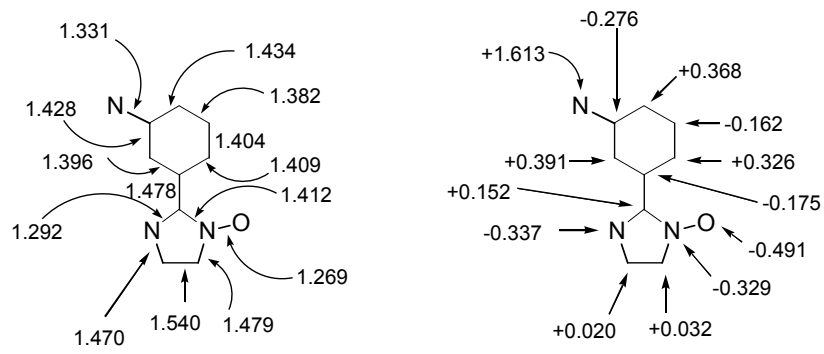

${ }^{2}$ A state, UB3LYP/6-31G* optimized

$E=-587.0841203\left(<S^{2}>=1.84\right)$

$0.0 \mathrm{cal} / \mathrm{mol}$
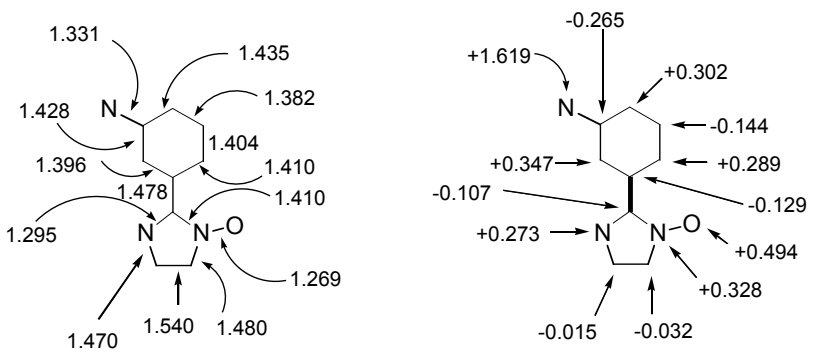

${ }^{4}$ A state, UB3LYP/6-31G* optimized

$E=-587.0830043\left(<S^{2}>=3.81\right)$

$+700 \mathrm{cal} / \mathrm{mol}$

Figure S1. UB3LYP/6-31G*//UB3LYP/6-31G* energies (hartrees), selected bond lengths (angstroms), and Mulliken spin density populations for quartet and doublet states of models for pNPhIN and mNPhIN. Methyl groups on the actual radical groups were replaced by hydrogen atoms for the computations. Relative energies are shown in cal/mol relative to the ground state for each system. The interfragment connection that leads to a spin density mismatch is shown in the ${ }^{2} A_{1}$ state of pNPhIN and the ${ }^{4} A$ state of mNPhIN. 
$1 \backslash 1 \backslash G I N C-G R O N D \backslash F O p t \backslash U B 3 L Y P \backslash 6-31 G$ (d) \C9H8N301 ( 4) \LAHTI \14-Jan-2005

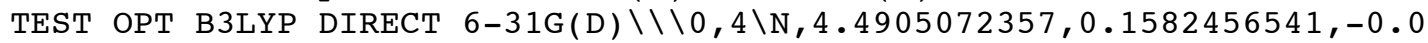
$013657675 \backslash \mathrm{C}, 3.172290342,0.1563264087,-0.0024005202 \backslash \mathrm{C}, 0.3326490279,0.14$ $58382331,-0.0042837084 \backslash \mathrm{C}, 2.4304576189,-1.075650039,0.0351625146 \backslash \mathrm{C}, 2.42$ $50908946,1.388353571,-0.0410868202 \backslash \mathrm{C}, 1.0519556521,1.3698858016,-0.0415$ $689941 \backslash \mathrm{C}, 1.0514507742,-1.0733244195,0.034005741 \backslash \mathrm{H}, 2.9844645598,-2.0080$ $608218,0.0644867247 \backslash \mathrm{H}, 2.9746204436,2.3234230577,-0.0696086164 \backslash \mathrm{H}, 0.4882$ $942802,2.2957596833,-0.0706332243 \backslash \mathrm{H}, 0.5060600142,-2.0070269116,0.06247$ $91884 \backslash \mathrm{C},-1.129035901,0.2249140681,-0.0078620572 \backslash 0,-1.6702481131,-2.126$ $7727555,0.0647766822 \backslash \mathrm{N},-1.9811291294,-0.8969010681,0.026308939 \backslash \mathrm{N},-1.77$ $43733513,1.3556771708,-0.0433972163 \backslash \mathrm{C},-3.3889599679,-0.4441438768,0.01$ $01210307 \backslash \mathrm{H},-3.8870005447,-0.8196029414,0.9090907884 \backslash \mathrm{C},-3.2179982895,1$. $0852745168,-0.0353287822 \backslash \mathrm{H},-3.6665239189,1.5851006947,0.8308875596 \backslash \mathrm{H},-$ $3.8834172516,-0.8724539196,-0.8669856362 \backslash \mathrm{H},-3.6669468701,1.5330453206$, $-0.929302352 \backslash \backslash$ Version=SGI64-G03RevB.03 \State $=4-\mathrm{A} \backslash \mathrm{HF}=-587.088008 \backslash \mathrm{S} 2=3.8$ $38013 \backslash \mathrm{S} 2-1=0 . \backslash \mathrm{S} 2 \mathrm{~A}=3.752854 \backslash \mathrm{RMSD}=5.364 \mathrm{e}-09 \backslash \mathrm{RMSF}=1.808 \mathrm{e}-05 \backslash \mathrm{Dipole}=-1.783$ $4461,0.3660919,-0.0128751 \backslash \mathrm{PG}=\mathrm{C} 01[\mathrm{X}(\mathrm{C} 9 \mathrm{H} 8 \mathrm{~N} 301)] \backslash \backslash @$

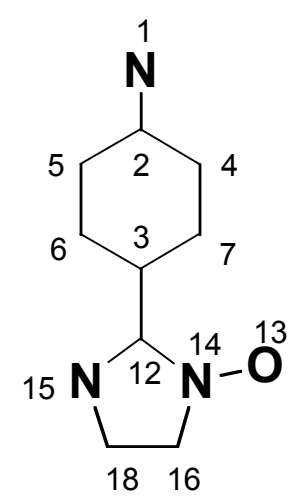

Mulliken atomic spin densities:

$$
\begin{array}{lr} 
& \multicolumn{1}{c}{1} \\
\mathrm{~N} & 1.546860 \\
\mathrm{C} & -0.283043 \\
\mathrm{C} & 0.338383 \\
\mathrm{C} & 0.357950 \\
\mathrm{C} & 0.325635 \\
\mathrm{C} & -0.173250 \\
\mathrm{C} & -0.178466 \\
\mathrm{H} & -0.013647 \\
\mathrm{H} & -0.012087 \\
\mathrm{H} & 0.008750 \\
\mathrm{H} & 0.007803 \\
\mathrm{C} & -0.180583 \\
\mathrm{O} & 0.491849 \\
\mathrm{~N} & 0.332698 \\
\mathrm{~N} & 0.407814 \\
\mathrm{C} & -0.032168 \\
\mathrm{H} & 0.023198 \\
\mathrm{C} & -0.024885 \\
\mathrm{H} & 0.016992 \\
\mathrm{H} & 0.023309 \\
\mathrm{H} & 0.016889
\end{array}
$$

Sum of Mulliken spin densities $=3.00000$ 


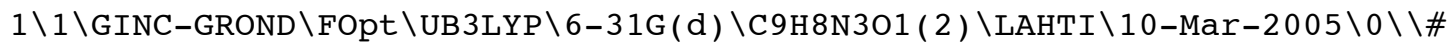
P UB3LYP/6-31G* GFINPUT IOP(6/7=3) TEST OPT \\Patrick Taylor doublet p-

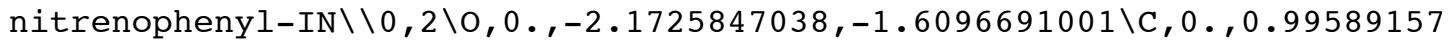
$56,-3.2509727461 \backslash \mathrm{C}, 0 .,-0.5395107935,-3.3793380102 \backslash \mathrm{N}, 0 ., 1.3051687163,-1$ $.8142245581 \backslash \mathrm{N}, 0$. $,-0.9528319079,-1.9595298542 \backslash \mathrm{C}, 0$. $0.1577322014,0.33340$ $80771 \backslash \mathrm{C}, 0 ., 0.2036955955,-1.1412503597 \backslash \mathrm{C}, 0 ., 0.2412145742,3.1668634151 \backslash \mathrm{C}$ $, 0.1 .3980014899,1.0167614317 \backslash \mathrm{C}, 0 .,-1.0404517729,1.0789876013 \backslash \mathrm{C}, 0 ., 1.4$ $520700314,2.3921398988 \backslash \mathrm{C}, 0$. $,-1.0073581523,2.4613752354 \backslash \mathrm{N}, 0 ., 0.27860366$ $44,4.4913757834 \backslash \mathrm{H}, 0$. , $2.3093382252,0.4289934472 \backslash \mathrm{H}, 0$. , $-1.9887374673,0.55$ $87445931 \backslash \mathrm{H}, 0 ., 2.4018544486,2.9166809763 \backslash \mathrm{H}, 0$. $,-1.9261818688,3.038371090$ $3 \backslash \mathrm{H}, 0.8806780219,1.4552399476,-3.714091705 \backslash \mathrm{H}, 0.8885544545,-0.955183704$ $2,-3.8642253768 \backslash \mathrm{H},-0.8806780219,1.4552399476,-3.714091705 \backslash \mathrm{H},-0.8885544$ $545,-0.9551837042,-3.8642253768 \backslash \backslash$ Version=SGI64-G03RevB.03 \State $=2-A^{\prime} \backslash H$ $\mathrm{F}=-587.0846075 \backslash \mathrm{S} 2=1.799113 \backslash \mathrm{S} 2-1=0 . \backslash \mathrm{S} 2 \mathrm{~A}=0.937788 \backslash \mathrm{RMSD}=7.521 \mathrm{e}-09 \backslash \mathrm{RMSF}=3$. $249 e-05 \backslash$ Dipole $=0 ., 0.2703932,-1.7100193 \backslash \mathrm{PG}=\mathrm{CS} \quad[\mathrm{SG}(\mathrm{C} 9 \mathrm{H} 4 \mathrm{~N} 301), \mathrm{X}(\mathrm{H} 4)] \backslash \backslash \mathrm{a}$

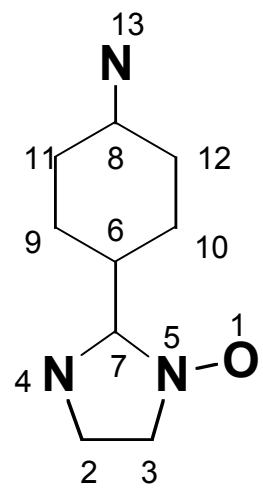

Mulliken atomic spin densities:

1

$\begin{array}{rrr}1 & \mathrm{O} & -0.446470 \\ 2 & \mathrm{C} & 0.019122 \\ 3 & \mathrm{C} & 0.026336 \\ 4 & \mathrm{~N} & -0.335188 \\ 5 & \mathrm{~N} & -0.280298 \\ 6 & \mathrm{C} & 0.300043 \\ 7 & \mathrm{C} & 0.112260 \\ 8 & \mathrm{C} & -0.271789 \\ 9 & \mathrm{C} & -0.144234 \\ 10 & \mathrm{C} & -0.140934 \\ 11 & \mathrm{C} & 0.326270 \\ 12 & \mathrm{C} & 0.349805 \\ 13 & \mathrm{~N} & 1.549777 \\ 14 & \mathrm{H} & 0.009068 \\ 15 & \mathrm{H} & 0.009355 \\ 16 & \mathrm{H} & -0.012088 \\ 17 & \mathrm{H} & -0.013158 \\ 18 & \mathrm{H} & -0.011109 \\ 19 & \mathrm{H} & -0.017830 \\ 20 & \mathrm{H} & -0.011109 \\ 21 & \mathrm{H} & -0.017830\end{array}$

Sum of Mulliken spin densities $=1.00000$ 
$1 \backslash 1 \backslash G I N C-G R O N D \backslash$ Stability \UB3LYP \6-31G (d) \C9H8N3O1 ( 4 ) \LAHTI $\backslash 21-M a r-2005$ $\backslash 0 \backslash \backslash \# P$ DIRECT 6-31G(D) GFINPUT IOP(6/7=3) UB3LYP TEST STABLE=OPT \\m-ni trenophenyl iminoylnitroxide $\backslash \backslash 0,4 \backslash \mathrm{C}, 0,-2.473887,1.710107,-0.076095 \backslash \mathrm{C}, 0$ $,-1.07893,1.55591,-0.055109 \backslash \mathrm{C}, 0,-0.511886,0.266365,-0.014595 \backslash \mathrm{C}, 0,-1.34$ $4244,-0.853009,0.004473 \backslash \mathrm{C}, 0,-2.764208,-0.707208,-0.01659 \backslash \mathrm{C}, 0,-3.31641$, $0.615295,-0.057791 \backslash \mathrm{C}, 0,0.949072,0.017852,0.009603 \backslash \mathrm{N}, 0,1.916005,1.04652$ $3,-0.005272 \backslash \mathrm{C}, 0,3.266099,0.442582,0.026796 \backslash \mathrm{C}, 0,2.927769,-1.059596,0.06$ $1488 \backslash \mathrm{N}, 0,1.461833,-1.166925,0.0465 \backslash 0,0,1.739056,2.302813,-0.039794 \backslash \mathrm{N}, 0$ $,-3.556305,-1.778293,0.001709 \backslash \mathrm{H}, 0,-2.893346,2.711809,-0.107319 \backslash \mathrm{H}, 0,-0$. $432979,2.422604,-0.069843 \backslash \mathrm{H}, 0,-0.91378,-1.846888,0.035617 \backslash \mathrm{H}, 0,-4.39569$ $, 0.727027,-0.073826 \backslash \mathrm{H}, 0,3.792314,0.811247,0.912536 \backslash \mathrm{H}, 0,3.813997,0.7653$ $44,-0.863698 \backslash \mathrm{H}, 0,3.31104,-1.555917,0.960051 \backslash \mathrm{H}, 0,3.332347,-1.601369,-0$. $800746 \backslash \backslash$ Version=SGI64-G03RevB.03 $\backslash$ State $=4-A \backslash H F=-587.0830043 \backslash \mathrm{S} 2=3.806452$ $\backslash \mathrm{S} 2-1=0 . \backslash \mathrm{S} 2 \mathrm{~A}=3.751315 \backslash \mathrm{RMSD}=9.675 \mathrm{e}-09 \backslash \mathrm{Dipole}=1.2425944,0.5175282,0.0014$ $473 \backslash \mathrm{PG}=\mathrm{C} 01 \quad[\mathrm{X}(\mathrm{C} 9 \mathrm{H} 8 \mathrm{~N} 301)] \backslash \backslash @$

Mulliken atomic spin densities:

1

$$
\begin{array}{rr}
\text { C } & -0.144167 \\
\mathrm{C} & 0.289018 \\
\mathrm{C} & -0.129378 \\
\mathrm{C} & 0.347170 \\
\mathrm{C} & -0.265179 \\
\mathrm{C} & 0.308409 \\
\mathrm{C} & -0.107425 \\
\mathrm{~N} & 0.328377 \\
\mathrm{C} & -0.031651 \\
\mathrm{C} & -0.015408 \\
\mathrm{~N} & 0.273190 \\
\mathrm{O} & 0.494267 \\
\mathrm{~N} & 1.619355 \\
\mathrm{H} & 0.008398 \\
\mathrm{H} & -0.014142 \\
\mathrm{H} & -0.013358 \\
\mathrm{H} & -0.011755 \\
\mathrm{H} & 0.022994 \\
\mathrm{H} & 0.022988 \\
\mathrm{H} & 0.009147 \\
\mathrm{H} & 0.009150
\end{array}
$$

Sum of Mulliken spin densities $=3.00000$

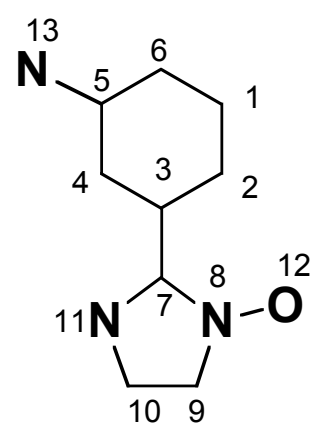


$1 \backslash 1 \backslash G I N C-G R O N D \backslash$ Stability \UB3LYP \6-31G(d) \C9H8N3O1 ( 2) \LAHTI \20-Mar-2005 $\backslash 0 \backslash \backslash \# P$ DIRECT 6-31G(D) GFINPUT IOP(6/7=3) UB3LYP TEST STABLE=OPT \\m-ni trenophenyl iminoylnitroxide doublet state $\backslash \backslash 0,2 \backslash \mathrm{C}, 0,-2.388163,1.49588$, $-0.068018 \backslash \mathrm{C}, 0,-0.993031,1.342596,-0.047075 \backslash \mathrm{C}, 0,-0.424194,0.052589,-0.0$ $06561 \backslash \mathrm{C}, 0,-1.256819,-1.068074,0.012521 \backslash \mathrm{C}, 0,-2.677452,-0.922704,-0.0085$ $18 \backslash \mathrm{C}, 0,-3.230431,0.400926,-0.049721 \backslash \mathrm{C}, 0,1.03333,-0.192942,0.017513 \backslash \mathrm{N}, 0$ , $2.0009,0.832582,0.002731 \backslash \mathrm{C}, 0,3.350862,0.22782,0.034844 \backslash \mathrm{C}, 0,3.012286,-$ $1.273839,0.069547 \backslash \mathrm{N}, 0,1.546598,-1.38119,0.054526 \backslash 0,0,1.826946,2.0892$,$0.031851 \backslash \mathrm{N}, 0,-3.468413,-1.992473,0.009747 \backslash \mathrm{H}, 0,-2.808142,2.497378,-0.09$ $9214 \backslash \mathrm{H}, 0,-0.346985,2.2093,-0.0618 \backslash \mathrm{H}, 0,-0.826173,-2.061745,0.043633 \backslash \mathrm{H}, 0$ $,-4.309725,0.512202,-0.065729 \backslash \mathrm{H}, 0,3.877083,0.596299,0.920602 \backslash \mathrm{H}, 0,3.898$ $771,0.550366,-0.855668 \backslash \mathrm{H}, 0,3.394936,-1.770654,0.968116 \backslash \mathrm{H}, 0,3.416301,-1$ $.816156,-0.792624 \backslash \backslash$ Version=SGI64-G03RevB.03\State=2-A \HF=-587.0841203\ $\mathrm{S} 2=1.83632 \backslash \mathrm{S} 2-1=0 . \backslash \mathrm{S} 2 \mathrm{~A}=1.060054 \backslash \mathrm{RMSD}=7.237 e-09 \backslash \mathrm{Dipole}=1.2503997,0.5019$ $264,0.002019 \backslash \mathrm{PG}=\mathrm{C} 01 \quad[\mathrm{X}(\mathrm{C} 9 \mathrm{H} 8 \mathrm{~N} 301)] \backslash \backslash @$

Mulliken atomic spin densities:

$$
1
$$

$$
\begin{array}{r}
-0.162318 \\
0.325879 \\
-0.174590 \\
0.390802 \\
-0.276391 \\
0.338965 \\
0.152425 \\
-0.329269 \\
0.032040 \\
0.019848 \\
-0.337327 \\
-0.491503 \\
1.613135 \\
0.009113 \\
-0.012566 \\
-0.013852 \\
-0.013133 \\
-0.022954 \\
-0.022949 \\
-0.012676 \\
-0.012678
\end{array}
$$

Sum of Mulliken spin densities $=1.00000$

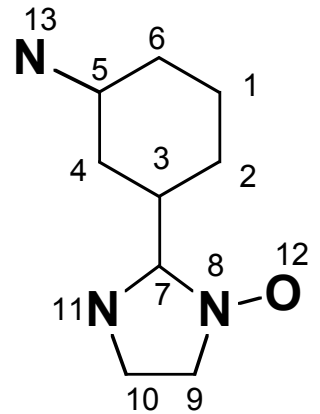


$1 \backslash 1 \backslash G I N C-G R O N D \backslash S P \backslash U T D-B 3 L Y P-F C \backslash 6-31 G(d) \backslash C 9 H 8 N 301$ ( 4 ) \LAHTI \30-Jun-2005 $0 \backslash \backslash \# P$ GFINPUT IOP $(6 / 7=3)$ TEST TD=NSTATES=5/6-31G* 5D UB3LYP GEOM=CHECK

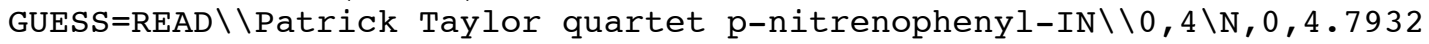
$59,0.048821,0.002277 \backslash \mathrm{C}, 0,3.475042,0.046902,0.001243 \backslash \mathrm{C}, 0,0.635401,0.036$ $414,-0.000641 \backslash \mathrm{C}, 0,2.73321,-1.185074,0.038806 \backslash \mathrm{C}, 0,2.727843,1.278929,-0$. $037444 \backslash \mathrm{C}, 0,1.354708,1.260462,-0.037926 \backslash \mathrm{C}, 0,1.354203,-1.182749,0.037649$ $\backslash \mathrm{H}, 0,3.287217,-2.117485,0.06813 \backslash \mathrm{H}, 0,3.277372,2.213999,-0.065966 \backslash \mathrm{H}, 0,0$. $791046,2.186336,-0.06699 \backslash \mathrm{H}, 0,0.808812,-2.116451,0.066122 \backslash \mathrm{C}, 0,-0.826284$ $, 0.11549,-0.004219 \backslash 0,0,-1.367496,-2.236197,0.06842 \backslash \mathrm{N}, 0,-1.678377,-1.00$ $6325,0.029952 \backslash \mathrm{N}, 0,-1.471621,1.246253,-0.039754 \backslash \mathrm{C}, 0,-3.086208,-0.553568$ $, 0.013764 \backslash \mathrm{H}, 0,-3.584249,-0.929027,0.912734 \backslash \mathrm{C}, 0,-2.915246,0.97585,-0.03$ $1686 \backslash \mathrm{H}, 0,-3.363772,1.475677,0.834531 \backslash \mathrm{H}, 0,-3.580665,-0.981878,-0.863343$ $\backslash \mathrm{H}, 0,-3.364195,1.423621,-0.925659 \backslash \backslash$ Version=SGI64-G03RevB .03 $\backslash$ State $=4-\mathrm{A} \backslash$ $\mathrm{HF}=-587.075537 \backslash \mathrm{S} 2=3.838102 \backslash \mathrm{S} 2-1=0 . \backslash \mathrm{S} 2 \mathrm{~A}=3.752858 \backslash \mathrm{RMSD}=6.893 e-09 \backslash \mathrm{PG}=\mathrm{C} 01$

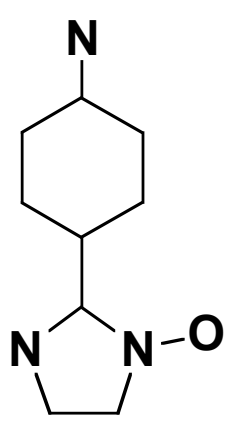
$[\mathrm{X}(\mathrm{C} 9 \mathrm{H} 8 \mathrm{~N} 301)] \backslash \backslash \mathrm{Q}$

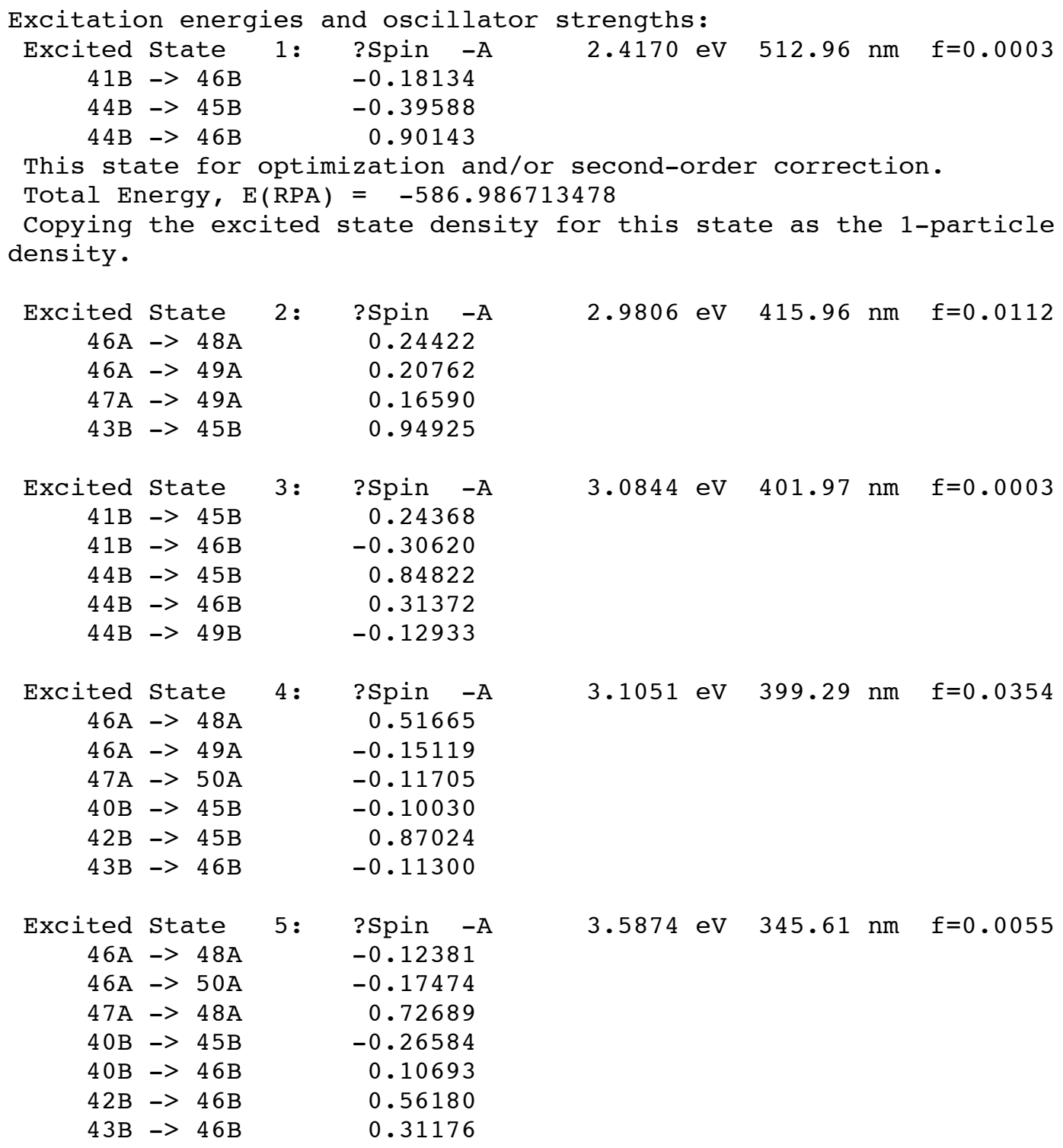


$1 \backslash 1 \backslash G I N C-G R O N D \backslash S P \backslash U T D-B 3 L Y P-F C \backslash 6-31 G(d) \backslash C 9 H 8 N 301$ ( 2) \LAHTI $01-J u 1-2005 \backslash$ $0 \backslash \backslash \# P$ GFINPUT IOP $(6 / 7=3)$ TEST TD=NSTATES=5/6-31G* 5D UB3LYP GEOM=CHECK GUESS=READ \\Patrick Taylor doublet p-nitrenophenyl-IN $\backslash \backslash 0,2 \backslash 0,0,0 .,-2$. $1725847038,-1.6096691001 \backslash \mathrm{C}, 0,0$. $0.9958915756,-3.2509727461 \backslash \mathrm{C}, 0,0 .,-0.5$ $395107935,-3.3793380102 \backslash \mathrm{N}, 0,0$. , $1.3051687163,-1.8142245581 \backslash \mathrm{N}, 0,0 .,-0.95$ $28319079,-1.9595298542 \backslash \mathrm{C}, 0,0.0 .1577322014,0.3334080771 \backslash \mathrm{C}, 0,0 ., 0.20369$ $55955,-1.1412503597 \backslash \mathrm{C}, 0,0$. , $0.2412145742,3.1668634151 \backslash \mathrm{C}, 0,0$. , 1.39800148 $99,1.0167614317 \backslash \mathrm{C}, 0,0 \ldots,-1.0404517729,1.0789876013 \backslash \mathrm{C}, 0,0 ., 1.4520700314$, $2.3921398988 \backslash \mathrm{C}, 0,0 \ldots,-1.0073581523,2.4613752354 \backslash \mathrm{N}, 0,0$. $0.2786036644,4.4$

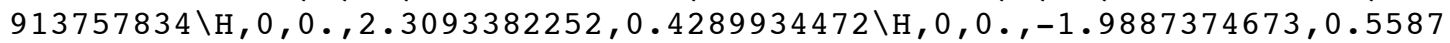
$445931 \backslash \mathrm{H}, 0,0$. , $2.4018544486,2.9166809763 \backslash \mathrm{H}, 0,0$. $,-1.9261818688,3.0383710$ $903 \backslash \mathrm{H}, 0,0.8806780219,1.4552399476,-3.714091705 \backslash \mathrm{H}, 0,0.8885544545,-0.955$ $1837042,-3.8642253768 \backslash \mathrm{H}, 0,-0.8806780219,1.4552399476,-3.714091705 \backslash \mathrm{H}, 0$, $-0.8885544545,-0.9551837042,-3.8642253768 \backslash \backslash$ Version=SGI64-G03RevB.03\St ate $=2-A^{\prime} \backslash \mathrm{HF}=-587.0721392 \backslash \mathrm{S} 2=1.799139 \backslash \mathrm{S} 2-1=0 . \backslash \mathrm{S} 2 \mathrm{~A}=0.937916 \backslash \mathrm{RMSD}=6.928 \mathrm{e}-$ $09 \backslash \mathrm{PG}=\mathrm{CS} \quad[\mathrm{SG}(\mathrm{C} 9 \mathrm{H} 4 \mathrm{~N} 3 \mathrm{O} 1), \mathrm{X}(\mathrm{H} 4)] \backslash \backslash \mathrm{a}$
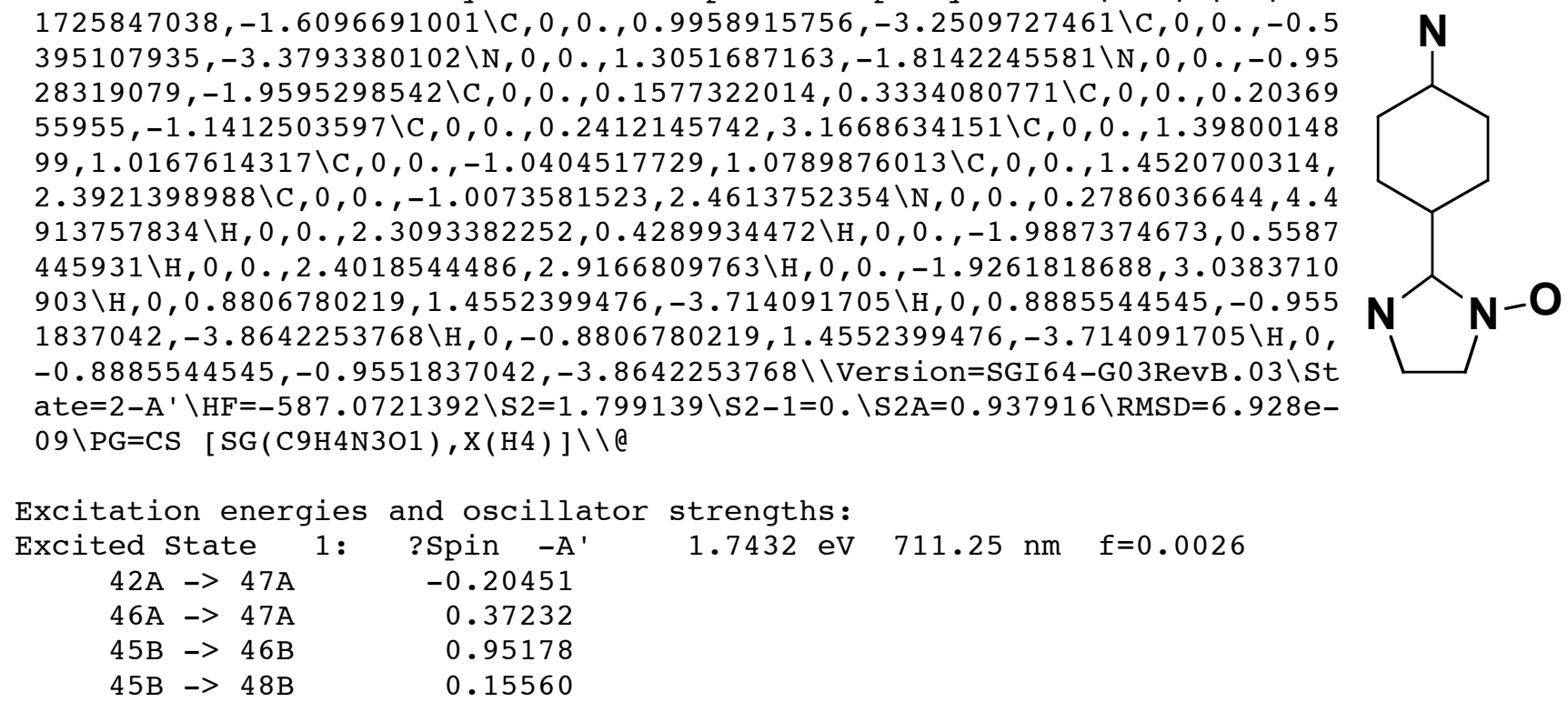

This state for optimization and/or second-order correction.

Total Energy, E(RPA) = -587.008078653

Copying the excited state density for this state as the 1-particle RhoCI density.

\begin{tabular}{|c|c|c|c|c|c|c|c|c|}
\hline Excited & State & $2:$ & ?Spin -A" & 2.3495 & $\mathrm{eV}$ & 527.70 & $\mathrm{~nm}$ & $f=0.0001$ \\
\hline $41 \mathrm{~A}$ & $->47 \mathrm{~A}$ & & 0.22711 & & & & & \\
\hline $44 \mathrm{~A}$ & $\rightarrow \quad 47 \mathrm{~A}$ & & 0.25781 & & & & & \\
\hline $45 \mathrm{~A}$ & $\rightarrow \quad 47 \mathrm{~A}$ & & 0.93711 & & & & & \\
\hline $45 \mathrm{~A}$ & $->48 \mathrm{~A}$ & & -0.12452 & & & & & \\
\hline Excited & State & $3:$ & ?Spin $\quad-A^{\prime}$ & 2.5280 & eV & 490.44 & $\mathrm{~nm}$ & $f=0.0153$ \\
\hline $46 \mathrm{~A}$ & $->47 \mathrm{~A}$ & & 0.89561 & & & & & \\
\hline $42 \mathrm{~B}$ & $->46 \mathrm{~B}$ & & -0.24080 & & & & & \\
\hline $45 B$ & $->46 B$ & & -0.30810 & & & & & \\
\hline $45 B$ & $\rightarrow 48 B$ & & 0.15191 & & & & & \\
\hline Excited & State & $4:$ & ?Spin & 2.8885 & $\mathrm{eV}$ & 429.23 & $\mathrm{~nm}$ & $f=0.0083$ \\
\hline $46 \mathrm{~A}$ & $->48 A$ & & 0.10314 & & & & & \\
\hline $46 \mathrm{~A}$ & $\rightarrow 49 A$ & & -0.30901 & & & & & \\
\hline $44 \mathrm{~B}$ & $->46 \mathrm{~B}$ & & 0.96807 & & & & & \\
\hline Excited & State & $5:$ & ?Spin -A" & 3.1881 & eV & 388.90 & $\mathrm{~nm}$ & $\mathrm{f}=0.0000$ \\
\hline $45 B$ & $->47 B$ & & 0.99910 & & & & & \\
\hline
\end{tabular}


$1 \backslash 1 \backslash G I N C-G R O N D \backslash S P \backslash U T D-B 3 L Y P-F C \backslash 6-31 G(d) \backslash C 9 H 8 N 301$ ( 4 ) \LAHTI \30-Jun-2005 $0 \backslash \backslash \# P$ GFINPUT IOP $(6 / 7=3)$ TEST TD=NSTATES=5/6-31G* 5D UB3LYP GEOM=CHECK GUESS=READ \\Patrick Taylor quartet p-nitrenophenyl-IN $\backslash \backslash 0,4 \backslash N, 0,4.7932$ $59,0.048821,0.002277 \backslash \mathrm{C}, 0,3.475042,0.046902,0.001243 \backslash \mathrm{C}, 0,0.635401,0.036$ $414,-0.000641 \backslash \mathrm{C}, 0,2.73321,-1.185074,0.038806 \backslash \mathrm{C}, 0,2.727843,1.278929,-0$. $037444 \backslash \mathrm{C}, 0,1.354708,1.260462,-0.037926 \backslash \mathrm{C}, 0,1.354203,-1.182749,0.037649$ $\backslash \mathrm{H}, 0,3.287217,-2.117485,0.06813 \backslash \mathrm{H}, 0,3.277372,2.213999,-0.065966 \backslash \mathrm{H}, 0,0$. $791046,2.186336,-0.06699 \backslash \mathrm{H}, 0,0.808812,-2.116451,0.066122 \backslash \mathrm{C}, 0,-0.826284$ $, 0.11549,-0.004219 \backslash 0,0,-1.367496,-2.236197,0.06842 \backslash \mathrm{N}, 0,-1.678377,-1.00$ $6325,0.029952 \backslash \mathrm{N}, 0,-1.471621,1.246253,-0.039754 \backslash \mathrm{C}, 0,-3.086208,-0.553568$ $, 0.013764 \backslash \mathrm{H}, 0,-3.584249,-0.929027,0.912734 \backslash \mathrm{C}, 0,-2.915246,0.97585,-0.03$ $1686 \backslash \mathrm{H}, 0,-3.363772,1.475677,0.834531 \backslash \mathrm{H}, 0,-3.580665,-0.981878,-0.863343$ $\backslash \mathrm{H}, 0,-3.364195,1.423621,-0.925659 \backslash \backslash$ Version=SGI64-G03RevB .03 \State $=4-\mathrm{A} \backslash$ $\mathrm{HF}=-587.075537 \backslash \mathrm{S} 2=3.838102 \backslash \mathrm{S} 2-1=0 . \backslash \mathrm{S} 2 \mathrm{~A}=3.752858 \backslash \mathrm{RMSD}=6.893 \mathrm{e}-09 \backslash \mathrm{PG}=\mathrm{C} 01$

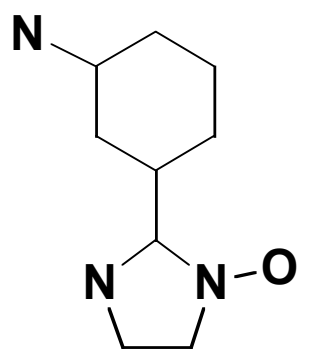
$[\mathrm{X}(\mathrm{C} 9 \mathrm{H} 8 \mathrm{~N} 301)] \backslash \backslash \mathrm{Q}$

$\begin{array}{rrr}\text { Excited State } & \text { 1: } & \text { ?Spin }-\mathrm{A} \\ 41 \mathrm{~B}-> & 46 \mathrm{~B} & -0.18134 \\ 44 \mathrm{~B}->45 \mathrm{~B} & -0.39588 \\ 44 \mathrm{~B}->46 \mathrm{~B} & 0.90143\end{array}$

This state for optimization and/or second-order correction.

Total Energy, E(RPA) = -586.986713478

Copying the excited state density for this state as the 1-particle RhoCI density.

\begin{tabular}{|c|c|c|c|c|c|c|c|c|}
\hline Excited & State & $2:$ & ?Spin $\quad-A$ & 2.9806 & eV & 415.96 & $\mathrm{~nm}$ & $f=0.0112$ \\
\hline $46 \mathrm{~A}$ & $->48 A$ & & 0.24422 & & & & & \\
\hline $46 \mathrm{~A}$ & $->49 A$ & & 0.20762 & & & & & \\
\hline $47 \mathrm{~A}$ & $->49 A$ & & 0.16590 & & & & & \\
\hline $43 B$ & $->45 B$ & & 0.94925 & & & & & \\
\hline Excited & State & $3:$ & ?Spin $\quad-A$ & 3.0844 & eV & 401.97 & $\mathrm{~nm}$ & $f=0.0003$ \\
\hline $41 \mathrm{~B}$ & $->45 B$ & & 0.24368 & & & & & \\
\hline $41 \mathrm{~B}$ & $->46 \mathrm{~B}$ & & -0.30620 & & & & & \\
\hline $44 \mathrm{~B}$ & $->45 B$ & & 0.84822 & & & & & \\
\hline $44 B$ & $->46 \mathrm{~B}$ & & 0.31372 & & & & & \\
\hline $44 \mathrm{~B}$ & $->49 B$ & & -0.12933 & & & & & \\
\hline Excited & State & $4:$ & ?Spin $\quad-A$ & 3.1051 & eV & 399.29 & $\mathrm{~nm}$ & $f=0.0354$ \\
\hline $46 \mathrm{~A}$ & $->48 \mathrm{~A}$ & & 0.51665 & & & & & \\
\hline $46 \mathrm{~A}$ & $->49 A$ & & -0.15119 & & & & & \\
\hline $47 \mathrm{~A}$ & $->50 A$ & & -0.11705 & & & & & \\
\hline $40 B$ & $->45 B$ & & -0.10030 & & & & & \\
\hline $42 B$ & $->45 B$ & & 0.87024 & & & & & \\
\hline $43 B$ & $->46 B$ & & -0.11300 & & & & & \\
\hline Excited & State & $5:$ & ?Spin $\quad-A$ & 3.5874 & eV & 345.61 & $\mathrm{~nm}$ & $f=0.0055$ \\
\hline $46 \mathrm{~A}$ & $->48 A$ & & -0.12381 & & & & & \\
\hline $46 \mathrm{~A}$ & $->50 A$ & & -0.17474 & & & & & \\
\hline $47 \mathrm{~A}$ & $->48 A$ & & 0.72689 & & & & & \\
\hline $40 B$ & $->45 B$ & & -0.26584 & & & & & \\
\hline $40 B$ & $->46 B$ & & 0.10693 & & & & & \\
\hline $42 B$ & $->46 B$ & & 0.56180 & & & & & \\
\hline $43 B$ & $->46 B$ & & 0.31176 & & & & & \\
\hline
\end{tabular}

Leave Link 914 at Thu Jun 30 21:56:39 2005, MaxMem= 6291456 cpu: 17066.8 
$1 \backslash 1 \backslash G I N C-G R O N D \backslash S P \backslash U T D-B 3 L Y P-F C \backslash 6-31 G(d) \backslash C 9 H 8 N 301$ ( 2) \LAHTI \05-Jul-2005 $0 \backslash \backslash \# P$ GEOM=CHECK DIRECT GFINPUT IOP $(6 / 7=3)$ TD=NSTATES=5/6-31G* 5D UB3L YP GUESS=READ \\m-nitrenophenyl iminoylnitroxide doublet state $\backslash \backslash 0,2 \backslash C, 0$ $,-2.3884221223,1.4956978142,-0.0680037866 \backslash \mathrm{C}, 0,-0.9932896659,1.34241433$ $95,-0.0470608101 \backslash \mathrm{C}, 0,-0.4244525204,0.0524075491,-0.0065466719 \backslash \mathrm{C}, 0,-1.2 \mathrm{~N}$ $570774707,-1.0682555146,0.012535239 \backslash \mathrm{C}, 0,-2.6777108693,-0.922885564,-0$. $0085042624 \backslash \mathrm{C}, 0,-3.2306902837,0.4007445926,-0.0497066612 \backslash \mathrm{C}, 0,1.03307111$ $06,-0.1931241511,0.017527042 \backslash \mathrm{N}, 0,2.0006410889,0.8324005674,0.002744871$ $\backslash \mathrm{C}, 0,3.3506032802,0.2276386345,0.0348577661 \backslash \mathrm{C}, 0,3.012026752,-1.2740207$ $842,0.0695604846 \backslash \mathrm{N}, 0,1.5463391448,-1.3813717109,0.0545393915 \backslash 0,0,1.826$ $6875471,2.0890178715,-0.03183677 \backslash \mathrm{N}, 0,-3.4686723266,-1.9926545866,0.009$ $7609587 \backslash \mathrm{H}, 0,-2.8084008794,2.4971957858,-0.0991996542 \backslash \mathrm{H}, 0,-0.3472441156$ $, 2.2091177729,-0.0617859308 \backslash \mathrm{H}, 0,-0.8264315539,-2.061926998,0.043646565$ $8 \backslash \mathrm{H}, 0,-4.3099836463,0.51202075,-0.0657146778 \backslash \mathrm{H}, 0,3.8768245178,0.596117$ $615,0.9206163048 \backslash \mathrm{H}, 0,3.8985119226,0.5501844837,-0.8556545699 \backslash \mathrm{H}, 0,3.394$ $6766555,-1.77083576,0.9681301679 \backslash \mathrm{H}, 0,3.4160421112,-1.8163380069,-0.792$ $6106314 \backslash \backslash$ Version=SGI64-G03RevB.03 \State $=2-A \backslash H F=-587.0716517 \backslash S 2=1.83639$ $7 \backslash \mathrm{S} 2-1=0 . \backslash \mathrm{S} 2 \mathrm{~A}=1.06031 \backslash \mathrm{RMSD}=5.015 \mathrm{e}-09 \backslash \mathrm{PG}=\mathrm{C} 01 \quad[\mathrm{X}(\mathrm{C} 9 \mathrm{H} 8 \mathrm{~N} 301)] \backslash \backslash \mathrm{a}$

Excitation energies and oscillator strengths:

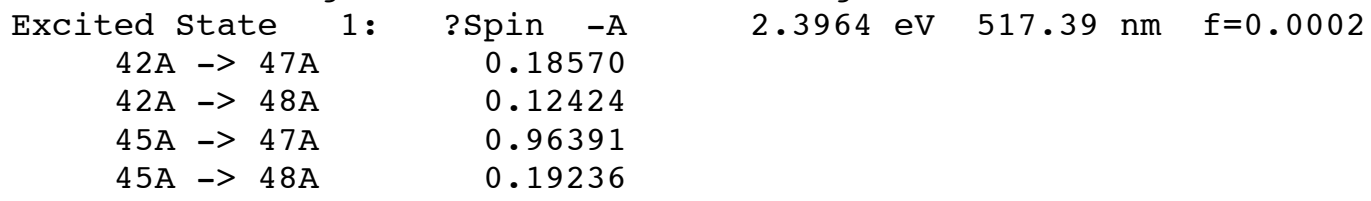

This state for optimization and/or second-order correction.

Total Energy, E(RPA) = -586.983587182

Copying the excited state density for this state as the 1-particle RhoCI density.

\begin{tabular}{|c|c|c|c|c|c|c|c|c|}
\hline $\begin{array}{r}\text { Excited } \\
45 \mathrm{~B}\end{array}$ & $\begin{array}{l}\text { State } \\
->46 \mathrm{~B}\end{array}$ & $2:$ & $\begin{array}{c}\text { ?Spin -A } \\
0.99865\end{array}$ & 2.4369 & eV & 508.78 & $\mathrm{~nm}$ & $f=0.0006$ \\
\hline $\begin{array}{r}\text { Excited } \\
46 \mathrm{~A} \\
46 \mathrm{~A} \\
44 \mathrm{~B}\end{array}$ & $\begin{array}{l}\text { State } \\
->47 \mathrm{~A} \\
->48 \mathrm{~A} \\
->46 \mathrm{~B}\end{array}$ & $3:$ & $\begin{array}{c}\text { ?Spin -A } \\
0.95432 \\
-0.16649 \\
-0.27187\end{array}$ & 2.6735 & $\mathrm{eV}$ & 463.75 & $\mathrm{~nm}$ & $f=0.0035$ \\
\hline $\begin{array}{r}\text { Excited } \\
41 \mathrm{~A} \\
44 \mathrm{~A} \\
46 \mathrm{~A} \\
46 \mathrm{~A} \\
46 \mathrm{~A} \\
44 \mathrm{~B} \\
44 \mathrm{~B} \\
45 \mathrm{~B}\end{array}$ & $\begin{array}{l}\text { State } \\
->47 \mathrm{~A} \\
->47 \mathrm{~A} \\
->47 \mathrm{~A} \\
->48 \mathrm{~A} \\
->49 \mathrm{~A} \\
->46 \mathrm{~B} \\
->48 \mathrm{~B} \\
->48 \mathrm{~B}\end{array}$ & $4:$ & $\begin{array}{l}\text { ?Spin -A } \\
0.12839 \\
0.39610 \\
0.18280 \\
0.36044 \\
-0.12945 \\
0.73346 \\
-0.14111 \\
-0.38175\end{array}$ & 3.1217 & $\mathrm{eV}$ & 397.17 & $\mathrm{~nm}$ & $f=0.0132$ \\
\hline $\begin{array}{r}\text { Excited } \\
41 \mathrm{~A} \\
44 \mathrm{~A} \\
44 \mathrm{~A} \\
46 \mathrm{~A} \\
46 \mathrm{~A} \\
44 \mathrm{~B} \\
44 \mathrm{~B} \\
45 \mathrm{~B}\end{array}$ & $\begin{array}{l}\text { State } \\
->47 \mathrm{~A} \\
->47 \mathrm{~A} \\
->48 \mathrm{~A} \\
->47 \mathrm{~A} \\
->48 \mathrm{~A} \\
->46 \mathrm{~B} \\
->48 \mathrm{~B} \\
->48 \mathrm{~B}\end{array}$ & $5:$ & $\begin{array}{l}\text { ?Spin -A } \\
0.16045 \\
0.61654 \\
-0.15224 \\
-0.21783 \\
-0.25343 \\
-0.47753 \\
-0.21182 \\
-0.52377\end{array}$ & 3.1299 & $\mathrm{eV}$ & 396.13 & $\mathrm{~nm}$ & $f=0.0029$ \\
\hline
\end{tabular}

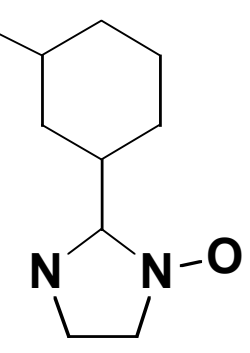


$1 \backslash 1 \backslash G I N C-G R O N D \backslash S P \backslash U T D-B 3 L Y P-F C \backslash 6-31 G(d) \backslash C 9 H 8 N 3 O 2$ ( 4 ) \LAHTI \07-Jul-2005 $0 \backslash \backslash \# P$ GFINPUT IOP $(6 / 7=3)$ TEST DIRECT TD=NSTATES=5/6-31G* 5D UB3LYP \\Bu rak quartet p-nitrenophenyl-NN $\backslash \backslash 0,4 \backslash 0,0,0.2 .339552,-1.453353 \backslash 0,0,0$. ,$2.339552,-1.453353 \backslash \mathrm{C}, 0,0.0 .763607,-3.229762 \backslash \mathrm{C}, 0,0,-0.763607,-3.22976$ $2 \backslash \mathrm{N}, 0,0 ., 1.11244,-1.789063 \backslash \mathrm{N}, 0,0 \ldots,-1.11244,-1.789063 \backslash \mathrm{C}, 0,0 \ldots, 0 ., 0.46039$ $6 \backslash \mathrm{C}, 0,0 ., 0 .,-0.978738 \backslash \mathrm{C}, 0,0 ., 0.3 .316108 \backslash \mathrm{C}, 0,0 ., 1.226514,1.192811 \backslash \mathrm{C}, 0$, 0 . , $-1.226514,1.192811 \backslash \mathrm{C}, 0,0$. , $1.232087,2.564584 \backslash \mathrm{C}, 0,0$. , $-1.232087,2.5645$ $84 \backslash \mathrm{N}, 0,0.0 ., 4.627562 \backslash \mathrm{H}, 0,0 ., 2.159348,0.646424 \backslash \mathrm{H}, 0,0 .,-2.159348,0.6464$ $24 \backslash \mathrm{H}, 0,0 ., 2.167421,3.114563 \backslash \mathrm{H}, 0,0 .,-2.167421,3.114563 \backslash \mathrm{H}, 0,0.888415,1.2$ $10526,-3.683141 \backslash \mathrm{H}, 0,0.888415,-1.210526,-3.683141 \backslash \mathrm{H}, 0,-0.888415,1.21052$ $6,-3.683141 \backslash \mathrm{H}, 0,-0.888415,-1.210526,-3.683141 \backslash \backslash$ Version=SGI $64-G 03$ RevB .0 $3 \backslash \mathrm{State}=4-\mathrm{A} 1 \backslash \mathrm{HF}=-662.2458265 \backslash \mathrm{S} 2=3.875793 \backslash \mathrm{S} 2-1=0 . \backslash \mathrm{S} 2 \mathrm{~A}=3.754903 \backslash \mathrm{RMSD}=4.6$

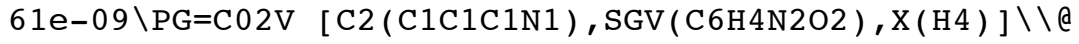

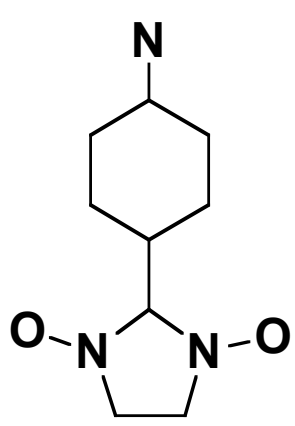

Excitation energies and oscillator strengths:

$\begin{array}{rlllll}\text { Excited State } & 1: & \text { ?Spin }-\mathrm{A} 1 & 2.6650 \mathrm{eV} & 465.23 \mathrm{~nm} & \mathrm{f}=0.0622 \\ \text { 50A } \rightarrow \text { 52A } & & 0.44872 & & \\ \text { 44B } \rightarrow \text { 49B } & 0.10768 & & \\ 48 \mathrm{~B} \rightarrow \text { 49B } & 0.91195 & & \end{array}$

This state for optimization and/or second-order correction.

Total Energy, E(RPA) = -662.147889767

Copying the excited state density for this state as the 1-particle RhoCI density.

\begin{tabular}{|c|c|c|c|c|c|c|}
\hline Excited & State & $2:$ & ?Spin $\quad-B 1$ & $2.7804 \mathrm{eV}$ & $445.92 \mathrm{~nm}$ & $\mathrm{f}=0.0001$ \\
\hline $45 B$ & $->49 B$ & & 0.49992 & & & \\
\hline $45 B$ & $->52 B$ & & 0.18683 & & & \\
\hline $47 B$ & $->50 \mathrm{~B}$ & & 0.85346 & & & \\
\hline Excited & State & $3:$ & ?Spin -A2 & $2.8327 \mathrm{eV}$ & $437.69 \mathrm{~nm}$ & $f=0.0000$ \\
\hline $45 B$ & $->50 \mathrm{~B}$ & & 0.65506 & & & \\
\hline $47 \mathrm{~B}$ & $->49 B$ & & 0.73424 & & & \\
\hline $47 \mathrm{~B}$ & $->52 B$ & & 0.20531 & & & \\
\hline Excited & State & $4:$ & ?Spin & $2.9330 \mathrm{eV}$ & $422.72 \mathrm{~nm}$ & $f=0.0051$ \\
\hline $51 \mathrm{~A}$ & $->52 \mathrm{~A}$ & & 0.74450 & & & \\
\hline $51 \mathrm{~A}$ & $->54 \mathrm{~A}$ & & 0.12510 & & & \\
\hline $46 B$ & $->49 B$ & & 0.12785 & & & \\
\hline $48 B$ & $->50 B$ & & -0.72831 & & & \\
\hline Excited & State & $5:$ & ?Spin & $3.0736 \mathrm{eV}$ & $403.38 \mathrm{~nm}$ & $f=0.0083$ \\
\hline $50 \mathrm{~A}$ & $->53 A$ & & 0.30473 & & & \\
\hline $51 \mathrm{~A}$ & $->52 \mathrm{~A}$ & & -0.10821 & & & \\
\hline $46 \mathrm{~B}$ & $->49 B$ & & 0.96018 & & & \\
\hline
\end{tabular}


$1 \backslash 1 \backslash G I N C-G R O N D \backslash S P \backslash U T D-B 3 L Y P-F C \backslash 6-31 G(d) \backslash C 9 H 8 N 301$ ( 2) \LAHTI $01-J u l-2005 \backslash$ $0 \backslash \backslash \# P$ GFINPUT IOP $(6 / 7=3)$ TEST TD=NSTATES=5/6-31G* 5D UB3LYP GEOM=CHECK GUESS=READ \\Patrick Taylor doublet p-nitrenophenyl-IN $\backslash \backslash 0,2 \backslash 0,0,0 .,-2$. $1725847038,-1.6096691001 \backslash \mathrm{C}, 0,0$, $0.9958915756,-3.2509727461 \backslash \mathrm{C}, 0,0 .,-0.5$ $395107935,-3.3793380102 \backslash \mathrm{N}, 0,0.1 .3051687163,-1.8142245581 \backslash \mathrm{N}, 0,0 .,-0.95$ $28319079,-1.9595298542 \backslash \mathrm{C}, 0,0.0 .1577322014,0.3334080771 \backslash \mathrm{C}, 0,0 ., 0.20369$ $55955,-1.1412503597 \backslash \mathrm{C}, 0,0$. , $0.2412145742,3.1668634151 \backslash \mathrm{C}, 0,0$. 1.39800148 $99,1.0167614317 \backslash \mathrm{C}, 0,0 \ldots,-1.0404517729,1.0789876013 \backslash \mathrm{C}, 0,0 ., 1.4520700314$, $2.3921398988 \backslash \mathrm{C}, 0,0 .,-1.0073581523,2.4613752354 \backslash \mathrm{N}, 0,0.0 .2786036644,4.4$

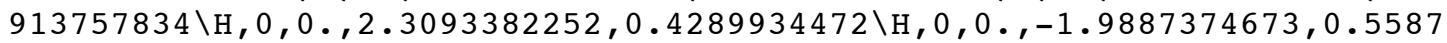
$445931 \backslash \mathrm{H}, 0,0 ., 2.4018544486,2.9166809763 \backslash \mathrm{H}, 0,0$. $,-1.9261818688,3.0383710$ $903 \backslash \mathrm{H}, 0,0.8806780219,1.4552399476,-3.714091705 \backslash \mathrm{H}, 0,0.8885544545,-0.955$ $1837042,-3.8642253768 \backslash \mathrm{H}, 0,-0.8806780219,1.4552399476,-3.714091705 \backslash \mathrm{H}, 0$, $-0.8885544545,-0.9551837042,-3.8642253768 \backslash \backslash$ Version=SGI64-G03RevB.03\St ate $=2-A^{\prime} \backslash \mathrm{HF}=-587.0721392 \backslash \mathrm{S} 2=1.799139 \backslash \mathrm{S} 2-1=0 . \backslash \mathrm{S} 2 \mathrm{~A}=0.937916 \backslash \mathrm{RMSD}=6.928 \mathrm{e}-$ $09 \backslash \mathrm{PG}=\mathrm{CS} \quad[\mathrm{SG}(\mathrm{C} 9 \mathrm{H} 4 \mathrm{~N} 3 \mathrm{O} 1), \mathrm{X}(\mathrm{H} 4)] \backslash \backslash \mathrm{a}$

\begin{tabular}{|c|c|c|c|c|c|}
\hline Excited & State & $1:$ & ?Spin & $1.7432 \mathrm{eV}$ & $711.25 \mathrm{~nm} \quad f=0.0026$ \\
\hline $42 A$ & $->47 A$ & & -0.20451 & & \\
\hline $46 \mathrm{~A}$ & $->47 \mathrm{~A}$ & & 0.37232 & & \\
\hline $45 \mathrm{~B}$ & $->46 B$ & & 0.95178 & & \\
\hline $45 \mathrm{~B}$ & $->48 B$ & & 0.15560 & & \\
\hline
\end{tabular}

This state for optimization and/or second-order correction.

Total Energy, E(RPA) = -587.008078653

Copying the excited state density for this state as the 1-particle RhoCI density.

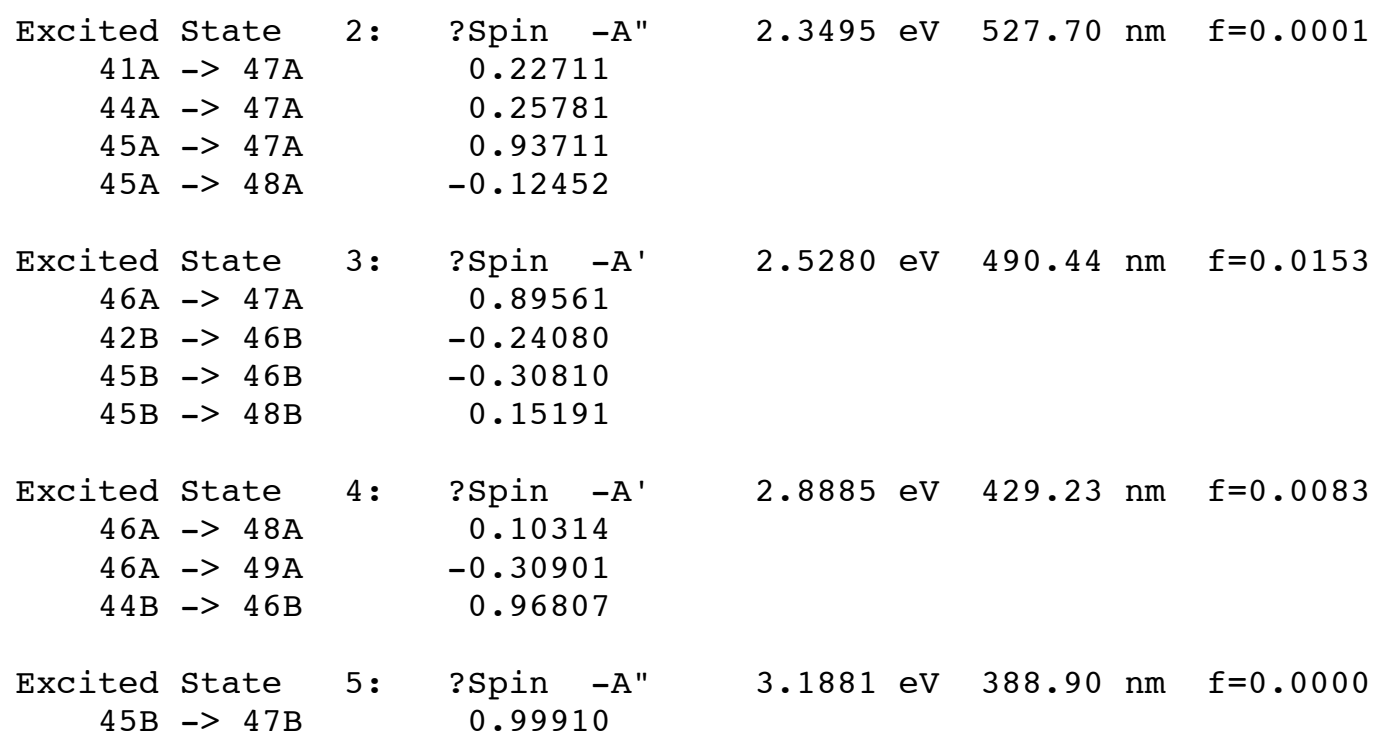

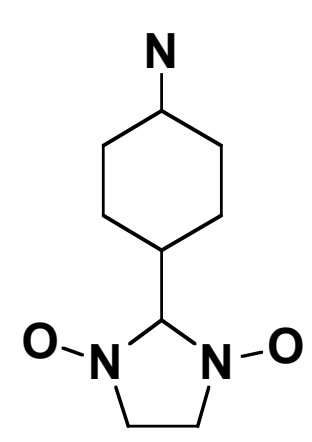


$1 \backslash 1 \backslash G I N C-G R O N D \backslash S P \backslash U T D-B 3 L Y P-F C \backslash 6-31 G(d) \backslash C 9 H 8 N 3 O 2$ ( 4) \LAHTI $\backslash 08-J u l-2005 \backslash$ $0 \backslash \backslash \# P$ GFINPUT IOP $(6 / 7=3)$ TEST TD=NSTATES=5/6-31G* 5D UB3LYP \\m-nitreno phenyl nitronylnitroxide $\backslash \backslash 0,4 \backslash \mathrm{C}, 0,-2.451006,1.710582,-0.173207 \backslash \mathrm{C}, 0,-1$. $064942,1.517604,-0.152049 \backslash \mathrm{C}, 0,-0.52713,0.214262,-0.019835 \backslash \mathrm{C}, 0,-1.39315$ $,-0.877306,0.091228 \backslash \mathrm{C}, 0,-2.809048,-0.681727,0.077007 \backslash \mathrm{C}, 0,-3.325764,0.6$ $4522,-0.060455 \backslash \mathrm{C}, 0,0.926994,0.012429,-0.002506 \backslash \mathrm{N}, 0,1.86379,0.998199,0$. $09756 \backslash \mathrm{C}, 0,3.245773,0.45618,0.084031 \backslash \mathrm{C}, 0,3.036141,-1.051914,-0.043014 \backslash \mathrm{N}$ $, 0,1.558721,-1.193084,-0.084631 \backslash 0,0,1.049128,-2.353061,-0.200124 \backslash 0,0,1$ $.69315,2.254939,0.210091 \backslash \mathrm{N}, 0,-3.633223,-1.723848,0.188921 \backslash \mathrm{H}, 0,-2.84022$ $7,2.719424,-0.278341 \backslash \mathrm{H}, 0,-0.393174,2.360767,-0.22933 \backslash \mathrm{H}, 0,-1.005296,-1$. $881887,0.181734 \backslash \mathrm{H}, 0,-4.401544,0.787122,-0.073672 \backslash \mathrm{H}, 0,3.733302,0.765205$ $, 1.011927 \backslash \mathrm{H}, 0,3.774581,0.908223,-0.758796 \backslash \mathrm{H}, 0,3.4039,-1.629402,0.80876$ $3 \backslash \mathrm{H}, 0,3.438028,-1.485326,-0.962172 \backslash \backslash$ Version=SGI64-G03RevB. 03 $\backslash$ State $=4-\mathrm{A}$ $\backslash \mathrm{HF}=-662.236685 \backslash \mathrm{S} 2=3.832711 \backslash \mathrm{S} 2-1=0 . \backslash \mathrm{S} 2 \mathrm{~A}=3.75258 \backslash \mathrm{RMSD}=7.278 \mathrm{e}-09 \backslash \mathrm{PG}=\mathrm{C} 01$ $[\mathrm{X}(\mathrm{C} 9 \mathrm{H} 8 \mathrm{~N} 3 \mathrm{O} 2)] \backslash \backslash \mathrm{Q}$

\begin{tabular}{|c|c|c|c|}
\hline Excited & tate & $1:$ & ?Spin \\
\hline $51 \mathrm{~A}$ & $->52 A$ & & 0.85750 \\
\hline $51 \mathrm{~A}$ & $->54 \mathrm{~A}$ & & 0.13107 \\
\hline $48 B$ & $->50 B$ & & -0.69260 \\
\hline
\end{tabular}

This state for optimization and/or second-order correction.

Total Energy, E(RPA) = -662.159951014

Copying the excited state density for this state as the 1-particle RhoCI density.

\begin{tabular}{|c|c|c|c|c|c|c|c|c|}
\hline Excited & State & $2:$ & ?Spin $-A$ & 2.3058 & $\mathrm{eV}$ & 537.70 & $\mathrm{~nm}$ & $f=0.0010$ \\
\hline $50 \mathrm{~A}$ & $->52 \mathrm{~A}$ & & -0.45364 & & & & & \\
\hline $44 \mathrm{~B}$ & $->49 B$ & & 0.12417 & & & & & \\
\hline $45 B$ & $->49 B$ & & 0.10381 & & & & & \\
\hline $48 B$ & $->49 B$ & & 0.92480 & & & & & \\
\hline Excited & State & $3:$ & ?Spin $-A$ & 2.8102 & $\mathrm{eV}$ & 441.19 & $\mathrm{~nm}$ & $f=0.0001$ \\
\hline $46 \mathrm{~B}$ & $->52 B$ & & -0.21137 & & & & & \\
\hline $47 \mathrm{~B}$ & $->50 B$ & & 0.97613 & & & & & \\
\hline Excited & State & $4:$ & ?Spin $-A$ & 3.0883 & $\mathrm{eV}$ & 401.47 & $\mathrm{~nm}$ & $f=0.0000$ \\
\hline $45 B$ & $->50 B$ & & -0.19047 & & & & & \\
\hline $46 B$ & $->50 B$ & & 0.93538 & & & & & \\
\hline $47 \mathrm{~B}$ & $->52 B$ & & -0.28279 & & & & & \\
\hline Excited & State & $5:$ & ?Spin $-A$ & 3.2075 & $\mathrm{eV}$ & 386.54 & $\mathrm{~nm}$ & $f=0.0206$ \\
\hline $50 \mathrm{~A}$ & $->52 \mathrm{~A}$ & & 0.84773 & & & & & \\
\hline $44 \mathrm{~B}$ & $->49 B$ & & -0.25690 & & & & & \\
\hline $48 B$ & $->49 B$ & & 0.34804 & & & & & \\
\hline $48 B$ & $->52 B$ & & -0.14950 & & & & & \\
\hline
\end{tabular}

2.0880 eV $593.78 \mathrm{~nm} \quad \mathrm{f}=0.0019$

(1)

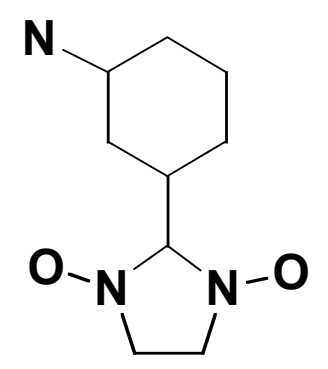


$1 \backslash 1 \backslash G I N C-G R O N D \backslash S P \backslash U T D-B 3 L Y P-F C \backslash 6-31 G(d) \backslash C 9 H 8 N 3 O 2$ ( 2) \LAHTI $09-J u 1-2005 \backslash$ $0 \backslash \backslash \# P$ GFINPUT IOP $(6 / 7=3)$ TEST TD=NSTATES=5/6-31G* 5D UB3LYP \\m-nitreno phenyl nitronylnitroxide radical state $\backslash \backslash 0,2 \backslash C, 0,-2.455536,1.718003,-0$. $047281 \backslash \mathrm{C}, 0,-1.069055,1.526532,-0.031333 \backslash \mathrm{C}, 0,-0.52537,0.215516,-0.00799$ $1 \backslash \mathrm{C}, 0,-1.395011,-0.883093,-0.001118 \backslash \mathrm{C}, 0,-2.812177,-0.688113,-0.017277 \backslash$ $\mathrm{C}, 0,-3.331141,0.647769,-0.040778 \backslash \mathrm{C}, 0,0.922199,0.01396,0.008617 \backslash \mathrm{N}, 0,1.8$ $67024,1.00253,0.006786 \backslash \mathrm{C}, 0,3.24741,0.458301,0.026856 \backslash \mathrm{C}, 0,3.035241,-1.0$ $53954,0.041221 \backslash \mathrm{N}, 0,1.558016,-1.196495,0.028585 \backslash 0,0,1.051245,-2.363248$, $0.036546 \backslash 0,0,1.704785,2.26513,-0.008794 \backslash \mathrm{N}, 0,-3.631072,-1.735604,-0.010$ $492 \backslash \mathrm{H}, 0,-2.84453,2.732321,-0.065091 \backslash \mathrm{H}, 0,-0.39907,2.374184,-0.036542 \backslash \mathrm{H}$, $0,-1.007754,-1.891555,0.016373 \backslash \mathrm{H}, 0,-4.40688,0.789294,-0.053005 \backslash \mathrm{H}, 0,3.7$ $47636,0.849486,0.916231 \backslash \mathrm{H}, 0,3.765887,0.830093,-0.860373 \backslash \mathrm{H}, 0,3.411171,-$ $1.551097,0.938811 \backslash \mathrm{H}, 0,3.428165,-1.570319,-0.838063 \backslash \backslash$ Version=SGI64-G03R evB.03 $\backslash$ State $=2-A \backslash H F=-662.2391026 \backslash \mathrm{S} 2=1.897485 \backslash \mathrm{S} 2-1=0 . \backslash \mathrm{S} 2 \mathrm{~A}=1.281899 \backslash \mathrm{RMSD}$ $=5.426 \mathrm{e}-09 \backslash \mathrm{PG}=\mathrm{C} 01[\mathrm{X}(\mathrm{C} 9 \mathrm{H} 8 \mathrm{~N} 3 \mathrm{O} 2)] \backslash \backslash \mathrm{a}$

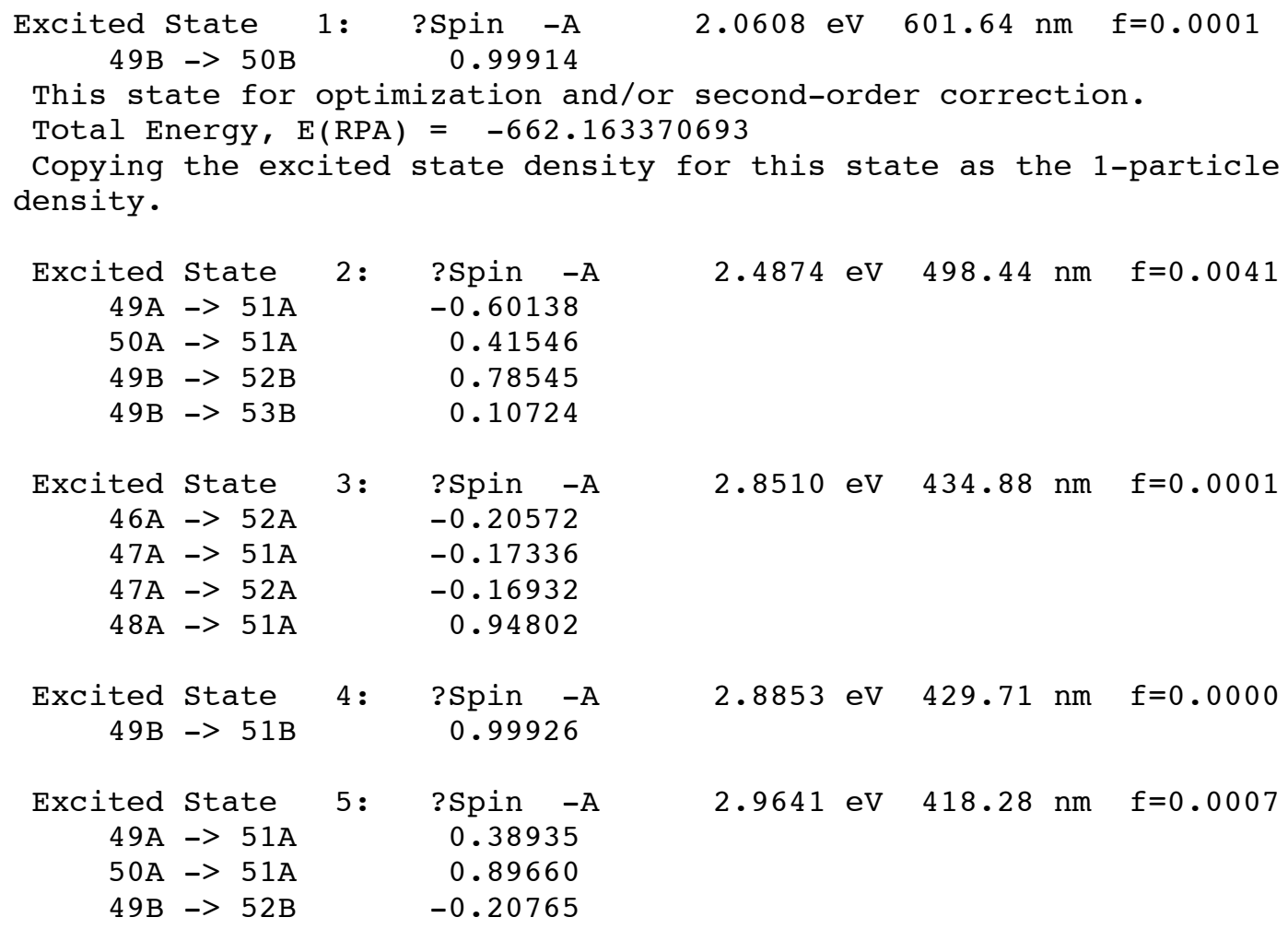

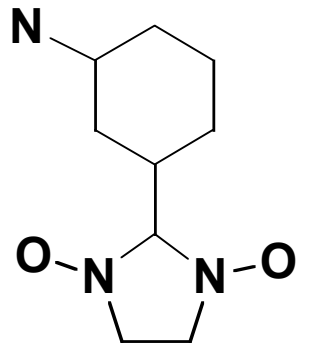

